\title{
Verzeichnis übernationaler und nationaler Quellen
}

\section{Staatsverträge}

Satzung des Europarates vom 5. Mai 1949 (Übersetzung aus dem französischen und englischen Originaltext), SR 0.192.030

Konvention vom 4. November 1950 zum Schutze der Menschenrechte und Grundfreiheiten, SR 0.101

Haager Übereinkommen vom 14. Mai 1954 für den Schutz von Kulturgut bei bewaffneten Konflikten, 249 UNTS 240; SR 0.520.3

Europäisches Kulturabkommen vom 19. Dezember 1954; ETS No. $18=$ SR 0.440 .1

Europäisches Übereinkommen vom 6. Mai 1969 zum Schutz archäologischen Kulturgutes; ETS Nr. 121; BGB1. 1974 II, 1285 ff.

Übereinkommen über Massnahmen zum Verbot und zur Verhütung der rechtswidrigen Einfuhr, Ausfuhr und Übereignung von Kulturgut vom 14. November 1970 (UNESCOKonvention 1970), 823 UNTS 231, ILM 10 (1971) 289; SR 0.444.1

Abkommen vom 22. Juli 1972 zwischen der Schweizerischen Eidgenossenschaft und der Europäischen Wirtschaftsgemeinschaft, SR 0.632.401

UNESCO-Konvention zum Schutz des Kultur- und Naturerbes der Welt vom 16. November 1972, ILM 11 (1972) 1358; SR 0.451.41

Übereinkommen zum Schutz des architektonischen Erbes Europas vom 3. Oktober 1985; ETS Nr. 66; SR 0.440.4

Europäisches Übereinkommen zum Schutz des archäologischen Erbes vom 16. Januar 1992, ETS No. $143=$ SR 0.440 .5

UNIDROIT-Übereinkommen über gestohlene oder rechtswidrig ausgeführte Kulturgüter vom 24. Juni 1995, ILM 34 (1995) 1330; SZIER 1997, 55 (fr.) und ZVglRWiss 95 (1996), 203 (engl.); deutsche Übersetzung in: Bundesamt für Kulturgut (Hrsg.), Internationaler Kulturgütertransfer, Bericht der Arbeitsgruppe, Bern 1998 (Unidroit-Konvention 1995)

Abkommen vom 21. Juni 1999 zwischen der Schweizerischen Eidgenossenschaft einerseits und der Europäischen Gemeinschaft und ihren Mitgliedstaaten andererseits über die Freizügigkeit; SR 0.142.112.681

\section{Europarechtliche Quellen und Materialien}

Vertrag zur Gründung der Europäischen Gemeinschaft vom 25. März 1957 [198 UNTS 11] i.d.F. des Vertrags von Amsterdam vom 10.11.1997, ABl. Nr. C 340/173 vom 10.11.1997

Richtlinie 64/221/EWG des Rates vom 25. Februar 1964 zur Koordinierung der Sondervorschriften für die Einreise und den Aufenthalt von Ausländern, soweit sie aus 
Gründen der öffentlichen Ordnung, Sicherheit oder Gesundheit gerechtfertigt sind, AB1. Nr. P 56/850 vom 4.4.1964

Verordnung Nr. 802/68/EWG des Rates vom 27. Juni 1968 über die gemeinsame Begriffsbestimmung für den Warenursprung, ABl. Nr. L 148/1 vom 28.6.1968

Verordnung (EWG) Nr. 1612/68/EWG des Rates vom 15. Oktober 1968 über die Freizügigkeit der Arbeitnehmer innerhalb der Gemeinschaft, AB1. Nr. L 257/2 vom 19.10 .1968

Richtlinie 68/360/EWG des Rates vom 15. Oktober 1968 zur Aufhebung der Reise- und Aufenthaltsbeschränkungen für Arbeitnehmer der Mitgliedstaaten und ihre Familienangehörigen innerhalb der Gemeinschaft, ABl. Nr. L 257/13 vom 19.10.1968

Verordnung (EWG) Nr. 1251/70/EWG der Kommission vom 29. Juni 1970 über das Recht der Arbeitnehmer, nach Beendigung einer Beschäftigung im Hoheitsgebiet eines Mitgliedstaats zu verbleiben, ABl. Nr. L 142/24 vom 30.06.1970

Richtlinie 72/194/EWG des Rates vom 18. Mai 1972 über die Erweiterung des Geltungsbereichs der Richtlinie vom 25. Februar 1964 zur Koordinierung der Sondervorschriften für die Einreise und den Aufenthalt von Ausländern, soweit sie aus Gründen der öffentlichen Ordnung, Sicherheit oder Gesundheit gerechtfertigt sind, auf die Arbeitnehmer, die von dem Recht, nach Beendigung einer Beschäftigung im Hoheitsgebiet eines Mitgliedstaats verbleiben zu können, Gebrauch machen, ABl. Nr. L 121/32 vom 26.5.1972

Richtlinie 73/148/EWG des Rates vom 21. Mai 1973 zur Aufhebung der Reise- und Aufenthaltsbeschränkungen für Staatsangehörige der Mitgliedstaaten innerhalb der Gemeinschaft auf dem Gebiet der Niederlassung und des Dienstleistungsverkehrs, ABl. Nr. L 172/14 vom 28.6.1973

Entschliessung des Europäischen Parlaments zum Schutz des europäischen Kulturguts vom 13. Mai 1974, ABl. 1974 Nr. C 62 vom 30.5.1974

Richtlinie 75/34/EWG des Rates vom 17. Dezember 1974 über das Recht der Staatsangehörigen eines Mitgliedstaats, nach Beendigung der Ausübung einer selbständigen Tätigkeit im Hoheitsgebiet eines anderen Mitgliedstaats zu verbleiben, ABl. Nr. L 14/10 vom 20.01.1975

Richtlinie 75/35/EWG des Rates vom 17. Dezember 1974 zur Erweiterung des Geltungsbereichs der Richtlinie 64/221/EWG zur Koordinierung der Sondervorschriften für die Einreise und den Aufenthalt von Ausländern, soweit sie aus Gründen der öffentlichen Ordnung, Sicherheit oder Gesundheit gerechtfertigt sind, auf die Staatsangehörigen eines Mitgliedstaats, die von dem Recht, nach Beendigung einer selbständigen Tätigkeit im Hoheitsgebiet eines Mitgliedstaats zu verbleiben, Gebrauch machen, ABl. Nr. L 14/14 vom 20.1.1975

Kommission der Europäischen Gemeinschaften, Mitteilung der Kommission an den Rat, „Die Aktion der Gemeinschaft im kulturellen Bereich“, Vorlage vom 22. November 1977, Bulletin der Europäischen Gemeinschaften, Beilage 6/77

Bericht des Ad-hoc-Ausschusses für das „Europa der Bürger“ (Adonnino-Ausschuss), „Ein Europa der Völker“ in: Bulletin der Europäischen Gemeinschaften, Beilage 7/1985

Einheitliche Europäische Akte vom 17./28. Februar 1986; ABl. L 169/1 vom 29.06. 1987 
Bericht der Kommission der Europäischen Gemeinschaft: Rapport sur la libre circulation des œuvres d'art dans la Communauté, KOM (1987) 1610 III B 1

Mitteilung der Kommission der Europäischen Gemeinschaft, an den Rat über den Schutz nationalen Kulturguts von künstlerischem, geschichtlichem oder archäologischem Wert im Hinblick auf die Beseitigung der Binnengrenzen im Jahre 1992; KOM (1989) 594 endg.

Richtlinie 90/364/EWG des Rates vom 28. Juni 1990 über das Aufenthaltsrecht, AB1. Nr. L 180/26 vom 13.7.1990

Richtlinie 90/365/EWG des Rates vom 28. Juni 1990 über das Aufenthaltsrecht der aus dem Erwerbsleben ausgeschiedenen Arbeitnehmer und selbständig Erwerbstätigen, ABl. Nr. L 180/28 vom 13.7.1990

Kommission der EG, Vorschlag für eine Verordnung (EWG) des Rates über die Ausfuhr von Kulturgütern, KOM (1991) 447 endg.

Vertrag über die Europäische Union vom 7. Februar 1992, ABl. Nr. C 191/1 vom 29.07. 1992

Verordnung 3911/92/EWG des Rates vom 9. Dezember 1992 über die Ausfuhr von Kulturgütern, AB1. Nr. L 395/1 vom 31.12.1992

Richtlinie 93/7/EWG des Rates vom 15. März 1993 über die Rückgabe von unrechtmässig aus dem Hoheitsgebiet eines Mitgliedstaats verbrachten Kulturgütern, AB1. Nr. L 74/74 vom 27.3.1993

Richtlinie 93/96/EWG des Rates vom 29. Oktober 1993 über das Aufenthaltsrecht der Studenten, AB1. Nr. L 317/59 vom 18.12.1993

Schlussfolgerungen des Rates vom 17. Juni 1994 zur Erstellung eines gemeinschaftlichen Aktionsplans im Bereich des kulturellen Erbes, ABl. Nr. C 235/1 vom 23. August 1994

Richtlinie 96/100/EG des Europäischen Parlaments und des Rates vom 17. Februar 1997 zur Änderung des Anhangs der Richtlinie 93/7/EWG über die Rückgabe von unrechtmässig aus dem Hoheitsgebiet eines Mitgliedstaats verbrachten Kulturgütern, ABl. Nr. L 60/59 vom 30.1.1997

Kommission der Europäischen Gemeinschaft, Zweiter Bericht der Kommission über die Unionsbürgerschaft Rapport, 27.05.1997, KOM (1997) 230 endg.

Beschluss Nr. 2228/97/EG des Europäischen Parlaments und des Rates vom 13. Oktober 1997 für ein Aktionsprogramm der Gemeinschaft zur Erhaltung des kulturellen Erbes - Programm „Raphael“, ABl. Nr. L 305/31 vom 8.11.1997

Beschluss Nr. 508/2000/EG des Europäischen Parlaments und des Rates vom 14. Februar 2000 über das Programm „Kultur 2000“, ABl. Nr. L 63/1 vom 10.3.2000

Charta der Grundrechte der Europäischen Union, ABl. Nr. C 364/1 vom 18.12.2000

Erläuterungen zu Art. 51 Grundrechtscharta, in: EuGRZ 2000, 559

Richtlinie 2001/38/EG des Europäischen Parlaments und des Rates vom 5. Juni 2001 zur Änderung der Richtlinie 93/7/EWG des Rates über die Rückgabe von unrechtmässig aus dem Hoheitsgebiet eines Mitgliedstaates verbrachten Kulturgütern, ABl. Nr. L 187/43 vom 10.7.2001 
Kommission der EG Vorschlag für einen Beschluss des Europäischen Parlaments und des Rates über das Programm „Kultur 2007“ (2007-2013), KOM (2004) 469 endg.

Richtlinie 2004/38/EG des Europäischen Parlaments und des Rates vom 29. April 2004 über das Recht der Unionsbürger und ihrer Familienangehörigen, sich im Hoheitsgebiet der Mitgliedstaaten frei zu bewegen und aufzuhalten, zur Änderung der Verordnung (EWG) Nr. 1612/68 und zur Aufhebung der Richtlinien 64/221/EWG, 68/360/EWG, 72/194/EWG, 73/148/EWG, 75/34/EWG, 75/35/EWG, 90/364/EWG, 90/ 365/EWG und 93/96/EWG, AB1. Nr. L 158/77 vom 30.4.2004

Beschluss Nr. 1855/2006/EG des Europäischen Parlaments und des Rates vom 12. Dezember 2006 über das Programm „Kultur“ (2007-2013), AB1. Nr. L 372/1, vom 27.12. 2006

\section{Nationale Quellen und Materialien}

\section{Italien}

Regio decreto del 30 gennaio 1913, n. 363, Regolamento di esecuzione delle Leggi 20 giugno 1909, n. 364, e 23 giugno 1912, n. 688, per le antichità e le belle arti, G.U. 5.6. 1913, n. 130

Legge del 1 giugno 1939, 1089, Tutela delle cose d'interesse artistico e storico [G.U. 8.8.1939, n. 184, 892; aufgehoben]

Codice civile, 16 marzo 1942, n. 262, G.U. 4.4.1942, n. 79-79bis

Costituzione della Repubblica italiana, 22 dicembre 1947, G.U. 27.12.1947, n. 298

Legge del 7 agosto 1990, n. 241, Nuove norme in materia di procedimento amministrativo e di diritto di accesso ai documenti amministrativi, G.U. 18.8.1990, n. 182

Legge 30 marzo 1998 n. 88, Norme sulla circolazione dei beni culturali, G.U. n. 84 vom 10.4.1998

Decreto legislativo del 29 ottobre 1999, n. 490, Testo unico delle diposizioni legislative in materia dei beni culturali e ambientali, a norma dell'art. 1 della legge 8 ottobre 1997, n. 352 [G.U. 27.12.1999, n. 302, aufgehoben]

Legge del 6 luglio 2002, n. 137, Delega per la riforma dell'organizzazione del Governo e della Presidenza del Consiglio dei Ministri, nonché di enti pubblici, GU 8.7.2002, n. 158

Decreto legislativo del 22 gennaio 2004, n. 42, recante il ,codice dei beni culturali e del paesaggio“", ai sensi dell'articolo 10 della legge 6 luglio 2002, n. 137, G.U. 24.2.2004, n. 45

Decreto del Presidente della Repubblica n. 173 dell'8 giugno 2004, Regolamento di organizzazione del Ministero per i beni e le attività culturali, G.U. 17.7.2004, n. 166

Decreto legislativo n. 156 del 24 marzo 2006, Disposizioni correttive ed integrative al decreto legislativo 22 gennaio 2004, n. 42, in relazione ai beni culturali, GU 27.4.2006 n. 97

Decreto legislativo n. 157 del 24 marzo 2006, Disposizioni correttive ed integrative al decreto legislativo 22 gennaio 2004, n. 42, in relazione al paesaggio, GU 27.4.2006 n. 97 


\section{Frankreich}

Loi du 31 décembre 1913 sur les monuments historiques, J. O. 4.1.1914

Loi du 31 décembre 1921 portant fixation du budget général de l'exercice 1922, J.O. 1.1.1922, 2

Loi du 27 septembre 1941 relative à la réglementation des fouilles archéologiques, J.O. 15.10.1941, 4438

Code du domaine de l'Etat, i.d.F. gemäss Décret 62-298 du 14 mars 1962 portant révision du code du domaine de l'Etat, J.O. 18.3.1962, 2897

Loi 92-1477 du 31 décembre 1992 relative aux produits soumis à certaines restrictions de circulation et à la complémentarité entre les services de police, de gendarmerie et de douane, J.O. 5.1.1993, 198

Décret 93-124 du 29 janvier 1993 relatif aux biens culturels soumis à certaines restrictions de circulation, J.O. 30.1.1993, 1600

Loi 95-877 du 3 août 1995 portant transposition de la directive 93/7 du 15 mars 1993 du Conseil des Communautés européennes relative à la restitution des biens culturels ayant quitté illicitement le territoire d'un Etat membre, J.O. 4.8.1995, 11664

Loi 2000-643 du 10 juillet 2000 relative à la protection des trésors nationaux et modifiant la Loi 92-1477 du 31 décembre 1992 relative aux produits soumis à certaines restrictions de circulation et à la complémentarité entre les services de police, de gendarmerie et de douane, J.O. 11.7.2000, 10481

\section{Spanien}

Ley 16/1985 del Patrimonio Histórico Español de 25 de junio de 1985; B.O.E. 29.6.1985, Nr. 155

Real Decreto 111/1986, de 10 de enero de desarrollo parcial de la Ley 16/1985, de 25 de junio, del Patrimonio Histórico Español, B.O.E. 28.1.1986

Ley 36/1994 de 23 de diciembre1994, de incorporación al ordinamiento juridico español de la Directiva 93/7/CEE del Consejo, de 15 de marzo 1993, relativa a la restitución de bienes que hayan salido de forma ilegal del territorio de un Estado miembro de la Unión Europea, B.O.E. 24.12.1994, no. 307, 38672; zuletzt geändert durch Ley 18/1998 de 15 de junio 1998, B.O.E. 16.6.1998, no. 143, 19799

\section{Deutschland}

Grundgesetz für die Bundesrepublik Deutschland vom 23. Mai 1949 [BGB1. 1949 I 1] i.d.F. des Gesetzes zur Änderung des Grundgesetzes vom 27. Oktober 1994, BGB1. 1994 I 3146; zuletzt geändert durch Gesetz vom 19. Dezember 2000, BGBl. I 1755

Amtliche Begründung des Regierungsentwurfs eines Kulturgutschutzgesetzes vom 19. November 1953, Deutscher Bundestag, 2. Wahlperiode 1953, BT-Drucksache 76/6

Gesetz zum Schutz deutschen Kulturgutes gegen Abwanderung vom 6. August 1955, BGB1. 1955 I 501 i.d.F. der Bekanntmachung der Neufassung vom 8. Juli 1999, BGB1. I S. 1754 
Empfehlung der Kultusministerkonferenz vom 3.12.1976 über die Ausdehnung des Gesamtverzeichnisses im Gesetz zum Schutz deutschen Kulturgutes gegen Abwanderung vom 6.8.1955, GMB1. 1977, 85

Kriterienkatalog vom 20. Mai 1983 zum Vollzug des Gesetzes zum Schutz deutschen Kulturgutes gegen Abwanderung vom 6. August 1995, GMB1. 1983, 442

Gesamtverzeichnisse national wertvollen Kulturgutes und national wertvoller Archive vom 19. April 1999, Bundesanzeiger Nr. 97a vom 28.5.1999

Gesetz zur Umsetzung von Richtlinien der Europäischen Gemeinschaften über die Rückgabe von unrechtmässig aus dem Hoheitsgebiet eines Mitgliedstaates verbrachten Kulturgütern und zur Änderung des Gesetzes zum Schutz deutschen Kulturgutes gegen Abwanderung (Kulturgutsicherungsgesetz) vom 15.10.1998, BGB1. 1998 I 3162

Gesetz zur Umsetzung der Richtlinie 93/7/EWG des Rates über die Rückgabe von unrechtmässig aus dem Hoheitsgebiet eines Mitgliedstaates verbrachten Kulturgütern (Kulturgüterrückgabegesetz) vom 15.10.1998, BGB1. 1998 I 3162

\section{Vereinigtes Königreich}

Import, Export and Customs Powers (Defence) Act 1939

Export of Goods (Control) Order 1992, S.I. 1992, 3092

The Return of Cultural Objects Regulations 1994, S.I. 1994, 501

Export Control Act 2002, 2002 Chapter 28, 24th July 2002

Explanatory Notes to Export Control Act 2002, 2002 Chapter 28

The Export of Objects of Cultural Interest (Control) Order 2003 (Export Order 2003), 18th November 2003, S. I. 2003, 2759

Open General Export Licence (Objects of Cultural Interest), 1st May 2004

Statutory guidance on the criteria to be taken into consideration when making a decision about whether or not to grant an export licence, Export Controls on Objects of Cultural Interest, November 2005

\section{Schweiz}

Schweizerisches Zivilgesetzbuch vom 10.12.1907, SR 210

Bundesgesetz vom 30.3.1911 betreffend die Ergänzung des Schweizerischen Zivilgesetzbuches (Fünfter Teil: Obligationenrecht), SR 220

Bundesverfassung der Schweizerischen Eidgenossenschaft vom 18. April 1999, SR 101

Bundesgesetz vom 20. Juni 2003 über den internationalen Kulturgütertransfer (Kulturgütertransfergesetz, KGTG), SR 444.1

Basel-Landschaft: $\S 4$ Abs. 1 Verordnung betreffend die Erhaltung von Altertümern vom 10.10.1921

Bern: Art. 11 Abs. 2 Gesetz über die Denkmalpflege vom 8.9.1999 und Art. 10 Abs. 1 i.V.m. Art. 37 Abs. 1 Verordnung über die Denkmalpflege vom 25.10.2000

Freiburg: Art. 24 Abs. 2 Gesetz über den Schutz der Kulturgüter vom 7.11.1991 
Graubünden: behördliche Praxis aufgrund Art. 1 Ziff. 4 Verordnung über den Naturund Heimatschutz vom 27.11.1946

Jura: Art. 5 Abs. 1 Loi sur la conservation des objets d'art et monuments historiques vom 9.11.1978

Luzern: 12 Gesetz über den Schutz der Kulturdenkmäler vom 8.3.1960

Nidwalden: Art. 53 Abs. 1 Gesetz über den Natur- und Heimatschutz vom 24.4.1988

Schwyz: $§ 7$ Verordnung betreffend den Natur- und Heimatschutz und die Erhaltung von Altertümern und Kunstdenkmälern vom 29.11.1927

St. Gallen: Art. 5 Abs. 2 Verordnung betreffend den Schutz von Naturkörpern und Altertümern vom 21.3.1933

Tessin: Art. 29 Abs. 1 Legge sulla protezione dei beni culturali vom 13.5.1997

\section{Rechtsprechung}

\section{Europäische Union}

EuGH 19.12.1961, Rs. 7/61 (Kommission der EWG gegen Regierung der Italienischen Republik), Slg. 1961, 695

EuGH 19.12.1968, Rs. 13/68 (Firma Salgoil gegen Aussenhandelsministerium der Italienischen Republik), Slg. 1968, 680

EuGH 12.10.1968, Rs. C 7/68 (Kommission der Europäischen Gemeinschaften gegen Italienische Republik), Slg. 1968, 634

EuGH 13.2.1969, Rs. 14/68 (Walt Wilhelm), Slg. 1969, 14

EuGH Rs. 11/70 (Internationale Handelsgesellschaft), Slg. 1970, 1125

EuGH 14.12.1972, Rs. 29/72 (S.P.A. Marinex gegen Italienische Finanzverwaltung), Slg. 1972, 1309

EuGH 12.7.1973, Rs. 2/73 (Riseria Luigi Geddo gegen Ente Nazionale Risi), Slg. 1973, 865

EuGH 12.2.1974, Rs. 152/73 (Giovanni Maria Sotgiu gegen Deutsche Bundespost), Slg. 1974,153

EuGH 14.5.1974, Rs. 4/73 (J. Nold, Kohlen- und Baustoffgrosshandlung gegen Kommission der Europaeischen Gemeinschaften), Slg. 1974, 491

EuGH 21.6.1974, Rs. 2/74 (Jean Reyners gegen Belgischen Staat), Slg. 1974, 631

EuGH 11.7.1974, Rs. 8/74 (Staatsanwaltschaft gegen Benoit und Gustave Dassonville), Slg. 1974, 837

EuGH 3.12.1974, Rs. 33/74 (Johannes Henricus Maria Van Binsbergen gegen Bestuur Van De Bedrijfsvereniging Voor De Metaalnijverheid), Slg. 1974, 1299

EuGH 4.12.1974, Rs. 41/74 (Yvonne Von Duyn gegen Home Office), Slg. 1974, 1337

EuGH 10.12.1974, Rs. 48/74 (Herr Charmasson gegen Minister für Wirtschaft und Finanzen), Slg. 1974, 1383 
EuGH 25.10.1975, Rs. 36/75 (Roland Rutili gegen Minister des Innern), Slg. 1975, 1219 EuGH 8.4.1976, Rs. 48/75 (Jean Noeel Royer), Slg. 1976, 497

EuGH 20.5.1976, Rs. 104/75 (Adriaan De Peijper, Geschäftsführer der Firma Centrafarm B.V.), Slg. 1976, 613

EuGH 7.7.1976, Rs. 118/75 (Lynne Watson und Alessandro Belmann), Slg. 1976, 1185

EuGH 15.12.1976, Rs. 35/76 (Simmenthal S.P.A. gegen Italienisches Finanzministerium), Slg. 1976, 1871

EuGH 25.1.1977, Rs. 46/76 (W.J.G. Bauhuis gegen niederländischen Staat), Slg. 1977, 5

EuGH 5.10.1977, Rs. 5/77 (Carlo Tedeschi gegen Denkavit Commerciale S.R.L.), Slg. 1977, 1555

EuGH 27.10.1977, Rs. 30/77 (Pierre Bouchereau), Slg. 1977, 1999

EuGH 12.10.1978, Rs. 13/78 (Joh. Eggers Sohn und Co. gegen Freie Hansestadt Bremen), Slg. 1978, 1935

EuGH 23.11.2978, 7/78 (Ernest George Thompson, Brian Albert Johnson und Colin Alex Norman Woodiwiss), Slg. 1978, 2247

EuGH 18.1.1979, Rs. 110 und 111/78 (Ministere Public und Chambre Syndicale des Agents Artistiques et Impresarii de Belgique, ASBL gegen Willy van Wesemael und Andere), Slg. 1979, 35

EuGH 20.2.1979, Rs. 120/78 (Rewe-Zentral AG gegen Bundesmonopolverwaltung für Branntwein), Slg. 1979, 649

EuGH 5.4.1979, Rs. 148/78 (Strafverfahren gegen Tullio Ratti), Slg. 1979, 1629

EuGH 12.7.1979, Rs. 153/78 (Kommission der Europäischen Gemeinschaften gegen Bundesrepublik Deutschland), Slg. 1979, 2555

EuGH 8.11.1979, Rs. 15/79 (P. B. Groenveld B.V. gegen Produktschap voor Vee en Vlees), Slg. 1979, 3409

EuGH 13.12.1979, Rs. 44/79 (Liselotte Hauer gegen Land Rheinland Pfalz), Slg. 1979, 3727

EuGH 14.12.1979 Rs. 34/79 (Strafverfahren gegen Maurice Donald Henn und John Frederick Ernest Darby), Slg. 1979, 3795

EuGH 17.12.1980, Rs. 149/79 (Kommission der Europäischen Gemeinschaften gegen Königreich Belgien), Slg. 1980, 3881

EuGH 26.2.1980, Rs. 94/79 (Strafverfahren gegen Pieter Vriend), Slg. 1980, 327

EuGH 18.3.1980, Rs. 52/79 (Strafverfahren gegen Marc J. V. C. Debauve und andere), Slg. 1980, 833

EUGH 17.6.1981, Rs. 113/80 (Kommission der Europäischen Gemeinschaften gegen Irland), Slg. 1981, 1625

EuGH 17.12.1981, Rs. 279/80 (Strafverfahren gegen Alfred John Webb), Slg. 1981, 3305

EuGH 9.6.1982, Rs. 95/81 (Kommission der Europäischen Gemeinschaften gegen Italienische Republik), Slg. 1982, 2187 
EuGH 18.5.1982, Rs. 115 und 116/81 (Rezguia Adoui gegen Belgischen Staat und Stadt Luettich - Dominique Cornuaille gegen Belgischen Staat), Slg. 1982, 1665

EuGH 9.6.1982, Rs. 95/81 (Kommission der Europäischen Gemeinschaften gegen Italienische Republik), Slg. 1982, 2187

EuGH 8.2.1983, Rs. 124/81 (Kommission der Europäischen Gemeinschaften gegen Vereinigtes Koenigreich Grossbritannien und Nordirland), Slg. 1983, 203

EuGH 13.3.1984, Rs. 16/83 (Starfverfahren gegen Prantl), Slg. 1984, 1299

EuGH 10.7.1984, Rs. 72/83 (Campus Oil und andere gegen Minister for Industry and Energy und andere), Slg. 1984, 2727

EuGH 6.9.1984, Rs. 177/83 (Firma Th. Kohl KG gegen Ringelhan und Rennett S.A. und Ringelhan Einrichtungs GmbH), Slg. 1984, 3651

EuGH 13.12.1984, Rs. 251/83 (Eberhard Haug-Adrion gegen Frankfurter Versicherungs-AG), Slg. 1984, 4277

EuGH 11.6.1985, Rs. 288/83 (Kommission der Europäischen Gemeinschaften gegen Irland), Slg. 1985, 1761

EuGH 11.7.1985, Rs. 60 und 64/84 (Société Cinéthèque S.A. und andere gegen Fédération nationale des cinémas français), Slg. 1985, 2605

EuGH 26.11.1985, Rs. 182/84 (Strafverfahren gegen Miro B.V.), Slg. 1985, 3731

EuGH 18.2.1986, Rs. 174/84 (Bulk Oil (Zug) AG gegen Sun International Limited und Sun Oil Trading Company), Slg. 1986, 559

EuGH 11.3.1986, Rs. 121/85 (Conegate Ltd gegen H. M. Customs and Excise), Slg. 1986, 1007

EuGH 30.4.1986, Rs. 96/85 (Kommission der Europäischen Gemeinschaften gegen Französische Republik), Slg. 1986, 1475

EuGH 3.6.1986, Rs. 307/84 (Kommission der Europäischen Gemeinschaften gegen Französische Republik), Slg. 1986, 1725

EuGH 3.7.1986, Rs. 66/85 (Deborah Lawrie-Blum gegen Land Baden-Württemberg), Slg. 1986, 2121

EuGH 10.7.1986, Rs. 79/85 (D. H. M. Segers gegen Bestuur van de Bedrijfsvereniging voor Bank- en Verzekeringswezen, Groothandel en Vrije Beroepen), Slg. 1986, 2375

EuGH 18.6.1987, Rs. 316/85 (Centre Public d'Aide Sociale de Courcelles gegen MarieChristine Lebon), Slg. 1987, 2811

EuGH 29.9.1987, Rs. 126/86 (Fernando Roberto Giménez Zaera gegen Instituto Nacional de la Seguridad Social et Tesoreria General de la Seguridad Social), Slg. 1987, 3697

EuGH 7.7.1988, Rs. 55/87 (Alexander Moksel Import und Export GmbH und Co. Handels-KG gegen Bundesanstalt für landwirtschaftliche Marktordnung), Slg. 1988, 3845

EuGH 27.9.1988, Rs. 81/87 (The Queen gegen H. M. Treasury und Commissioners of Inland Revenue, ex parte Daily Mail und General Trust Plc.), Slg. 1988, 5483

EUGH 18.5.1989, Rs. 249/86 (Kommission der Europaeischen Gemeinschaften gegen Bundesrepublik Deutschland), Slg. 1989, 1263 
EuGH 13.7.1989, Rs. 5/88 (Hubert Wachauf gegen Bundesrepublik Deutschland), Slg. 1989, 2609

EuGH 21.9.1989, Rs. 46/87 und 227/88 (Hoechst AG gegen Kommission der Europaeischen Gemeinschaften), Slg. 1989, 2859

EuGH 18.10.1990, Rs. C-297/88 u. C-197/89 (Massam Dzodzi gegen Etat Belge), Slg. 1990, I-3763

EuGH 12.12.1990, Rs. 302/88 (Hennen Olie BV gegen Stichting Interim Centraal Orgaan Voorraadvorming Aardolieprodukten und Niederlaendischer Staat), Slg. 1990, I-4625

EuGH 5.2.1991, Rs. C-363/89 (Danielle Roux gegen Belgischer Staat), Slg. 1991, I-273

EuGH 26.2.1991, Rs. C-154/89 (Kommission der Europäischen Gemeinschaften gegen Französische Republik), Slg. 1991, I-659

EuGH 26.2.1991, Rs. C-180/89 (Kommission der Europäischen Gemeinschaften gegen Italienische Republik), Slg. 1991, I-709

EuGH 26.2.1991, Rs. C-198/89 (Kommission der Europäischen Gemeinschaften gegen Republik Griechenland), Slg. 1991, I-727

EuGH 18.6.1991 Rs. C-260/89 (Elliniki Radiophonia Tileorassi Anonimi Etairia und Panellinia Omospondia Syllogon Prossopikou ERT Gegen Dimotiki Etairia Pliroforissis und Sotirios Kouvelas und Nicolaos Avdellas Und Andere), Slg. 1991, I-2925

EuGH 25.7.1991, Rs. C-288/89 (Stiching Collectieve Antennevoorziening Gouda und andere gegen Commissariaat voor De Media), Slg. 1991, I-4007

EuGH 4.10.1991, Rs. C-367/89 (Strafverfahren gegen Aime Richardt und Les Accessoires Scientifiques SNC), Slg. 1991, I-4621

EuGH 21.11.1991, Rs. C-27/91 (Union de recouvrement des cotisations de sécurité sociale et d'allocations familiales de la Savoie (URSSAF) contre Hostellerie Le Manoir SARL), Slg. 1991, I-5531

EuGH 8.4.1992, Rs. C-62/90 (Kommission der Europäischen Gemeinschaften gegen Bundesrepublik Deutschland), Slg. 1992, I-2575

EuGH 4.6.1992, Rs. C-360/90 (Arbeiterwohlfahrt der Stadt Berlin gegen Monika Bötel), Slg. 1992, I-3589

EuGH 7.7.1992, Rs. C-370/90 (The Queen gegen Immigration Appeal Tribunal und Surinder Singh, Ex Parte Secretary of State for Home Department), Slg. 1992, I-4265

EuGH 10.3.1993, Rs. C-111/91 (Kommission der Europäischen Gemeinschaften gegen Grossherzogtum Luxemburg), Slg. 1993, I-817

EuGH 31.3.1993, Rs. C-19/92 (Dieter Kraus GEGEN Land Baden-Württemberg), Slg. 1993, I-1663

EuGH 27.10.1993, Rs. C-127/92 (Dr. Pamela Mary Enderby gegen Frenchay Health Authority und Secretary of State for Health), Slg. 1993, I-5535

EuGH 24.11.1993, Rs. 267/91 u. 268/91 (Strafverfahren gegen Bernard Keck und Daniel Mithouard), Slg. 1993, I-6097

EuGH 24.3.1994, Rs. C-80/92 (Kommission der Europäischen Gemeinschaften gegen Königreich Belgien), Slg. 1994, I-1019 
EuGH 10.11.1994, Rs. C-320/93 (Lucien Ortscheit GmbH gegen Eurim-Pharm Arzneimittel GmbH), Slg. 1994, I-5243

EuGH 10.5.1995, Rs. C-384/93 (Alpine Investments BV gegen Minister van Financien), Slg. 1995, I-1141

EuGH 7.12.1995 Rs. C-449/93 (Rockfon A/S gegen Specialarbejderforbundet I Danmark) Slg. 1995, 4291

EuGH 13.7.1995, Rs. C-350/92 (Koenigreich Spanien gegen Rat Der Europäschen Union), Slg. 1995, I-1985

EuGH 30.11.1995, Rs. C-55/94 (Reinhard Gebhard gegen Consiglio dell'ordine degli Avvocati e Procuratori di Milano), Slg. 1995, I-4165

EuGH 15.12.1995, Rs. C-415/93 (Union Royale Belge des Sociétés de Football Association ASBL gegen Jean-Marc Bosman, Royal Club Liégeois SA gegen Jean-Marc Bosman und andere und Union des Associations Européennes de Football (UEFA) gegen Jean-Marc Bosman), Slg. 1995, I-4921

EuGH 15.2.1996, Rs. C-53/95 (Inasti (Institut national d'assurances sociales pour travailleurs indépendants) gegen Hans K), Slg. 1996, I-703

EuGH 23.5.1996, Rs. 237/94, (John O’Flynn gegen Adjudication Officer), Slg. 1996, I-2617

EuGH 20.6.1996, C-418/93 (Rs. Semeraro Casa Uno Srl gegen Sindaco del Comune di Erbusco), C-419/93 (Semeraro Mobili SpA gegen Sindaco del Comune di Erbusco), C-420/93 (RB Arredamento Srl gegen Sindaco del Comune di Stezzano), C-421/93 (Città Convenienza Milano Srl gegen Sindaco del Comune di Trezzano sul Naviglio), C-460/93 (Città Convenienza Bergamo Srl gegen Sindaco del Comune di Stezzano), C-461/93 (Centro Italiano Mobili Srl gegen Sindaco del Comune di Pineto), C-462/93 (Il 3C Centro Convenienza Casa Srl gegen Sindaco del Comune di Roveredo in Piano), C-464/93 (Benelli Confezioni SNC gegen Sindaco del Comune di Capena), C-9/94 (M. Quattordici Srl gegen Commissario straordinario del Comune di Terlizzi), C-10/94 (Società Italiana Elettronica Srl (SIEL) gegen Sindaco del Comune di Dozza), C-11/94 (Modaffari Srl gegen Sindaco del Comune di Trezzano sul Naviglio), C-14/94 (Modaffari Srl gegen Comune di Cinisello Balsamo), C-15/94 (Cologno Srl gegen Sindaco del Comune di Cologno Monzese), C-23/94 (Modaffari Srl gegen Sindaco del Comune di Osio Sopra), C-24/94 (M. Dieci Srl gegen Sindaco del Comune di Madignano) und C-332/94 (Consorzio Centro Commerciale „Il Porto“ gegen Sindaco del Comune di Adria), Slg. 1996, I-2975

EuGH 12.12.1996, Rs. C-3/95 (Reisebüro Broede gegen Gerd Sandker), Slg. 1996, I-6511

EuGH 9.12.1997, Rs. C-265/95 (Kommission der Europäischen Gemeinschaften gegen Französische Republik), Slg. 1997, I-6959

EuGH 13.5.1997, Rs. C-233/94 (Bundesrepublik Deutschland gegen Europäisches Parlament und Rat der Europäischen Union), Slg. 1997, I-2405

EuGH 15.5.1997, Rs. C-250/95 (Futura Participations SA und Singer gegen Administration des contributions), Slg. 1997, I-2471

EuGH 26.6.1997, Rs. C-368/95, (Vereinigte Familiapress Zeitungsverlags- und vertriebs GmbH gegen Heinrich Bauer Verlag), Slg. 1997, I-3689 
EuGH 9.12.1997, Rs. C-265/95 (Kommission der Europaeischen Gemeinschaften gegen Französische Republik), Slg. 1997, I-6959

EuGH 7.5.1998, Rs. C-350/96 (Clean Car Autoservice GesmbH gegen Landeshauptmann von Wien), Slg. 1998, I-2521

EuGH 12.5.1998, Rs. C-85/96 (María Martínez Sala gegen Freistaat Bayern), Slg. 1998, I-2691

EuGH 26.1.1999, Rs. C-18/95 (F.C. Terhoeve gegen Inspecteur van de Belastingdienst Particulieren/Ondernemingen buitenland), Slg. 1999, I-345

EuGH 11.5.1999, Rs. C-255/97 (Pfeiffer Grosshandel GmbH gegen Löwa Warenhandel $\mathrm{GmbH})$, Slg. 1999, I-2835

EuGH 27.1.2000, Rs. C-190/98 (Volker Graf gegen Filzmoser Maschinenbau GmbH), Slg. 2000, I-493

EuGH 11.4.2000, Rs. C-356/98 (Arben Kaba gegen Secretary of State for the Home Department), Slg. 2000, I-2623

EuGH 13.4.2000, Rs. C-176/96 (Jyri Lehtonen und Castors Canada Dry Namur-Braine ASBL gegen Fédération Royale Belge Des Sociétés De Basket-Ball Asbl (FRBSB)), Slg. 2000, I-2681

EuGH 1.2.2001, Rs. C-108/96 (Strafverfahren gegen Dennis Mac Quen, Derek Pouton, Carla Godts, Youssef Antoun und Grandvision Belgium SA, zivilrechtlich haftend, Beteiligte: Union professionnelle belge des médecins spécialistes en ophtalmologie et chirurgie oculaire), Slg. 2001, I-837

EuGH 17.9.2002, Rs. C-413/99 (Baumbast und R gegen Secretary of State for the Home Department), Slg. 2002, I-7091

EuGH 26.11.2002, Rs. C-100/01 (Ministre de l'Intérieur gegen Aitor Oteiza Olazabal), Slg. 2002, I-10981

EuGH 11.7.2002, Rs. C-224/98 (Marie-Nathalie D'Hoop gegen Office national de l'emploi), Slg. 2002, I-6191

EuGH 12.6.2003, Rs. 112/00 (Eugen Schmidberger, Internationale Transporte und Planzüge gegen Republik Österreich), Slg. 2003, I-5659

EuGH 9.7.2003, Rs. T-224/00 (Daniels Midland und Archer Daniels Midland Ingredients gegen Kommission der Europäischen Gemeinschaften), Slg. 2003, II-2597

EuGH 6.11.2003 Rs. C-101/01 (Strafverfahren gegen Bodil Lindqvist), Slg. 2003, I-12971

EuGH 1.4.2004, Rs. C-1/02 (Privat-Molkerei Borgmann GmbH \& Co. KG gegen Hauptzollamt Dortmund), Slg. 2004, I-3219

EuGH 11.3.2004, Rs. C 9/02 (Hughes de Lasteyrie du Saillant gegen Ministère de l'Économie, des Finances et de l'Industrie), Slg. 2004, 0000

GA Roemer, Schlussanträge in: EuGH Rs. $29 / 72$ (S.P.A. Marinex gegen Italienische Finanzverwaltung), Slg. 1972, 1309

GA La Pergola, Schlussanträge in: EuGH Rs. C-85/96 (María Martínez Sala gegen Freistaat Bayern), Slg. 1998, I-2691 
GA Mischo, Schlussanträge in: EuGH Rs. 249/86 (Kommission der Europaeischen Gemeinschaften gegen Bundesrepublik Deutschland), Slg. 1989, 1263

GA Mischo, Schlussanträge in: EuGH Rs. C-255/97 (Pfeiffer Grosshandel GmbH gegen Löwa Warenhandel GmbH), Slg. 1999, I-2835

\section{Italien}

Cass., sez.un., 6.4.1966, n. 898 (Finanze c. Chiesa parrocchiale di S. Stefano in Venezia; Chiesa parrocchiale di S. Stefano in Venezia c. Finanze), Foro it. 1966 I $2061=$ in Foro amm., 1966 I 346

Cons.Stato, sez. IV, 28.9.1967, n. 397 (Serra di Cassano c. Min. pubblica istruzione), Giust.civ. 1968 II 91

C.Cost., 26.4.1971, n. 79, Racc.Uff. 485

Cons.Stato, sez. VI, 13.3.1973, n. 199 (Cassa di risparmio di Trieste c. Min. pubblica istruzione), Foro it. 1973 III 259

Cons.Stato, ad.plen., 7.6.1973, n. 6 (Pres. Regione siciliana c. Soc. Cementerie siciliane), Foro it. 1973 III 408

Cass., sez.un., 3.5.1974, n. 1235 (Min. pubblica istruzione c. Vassallo), Foro it. 1974 I 2334

Cass., sez.un., 24.5.1975, n. 2102, Foro it. 1976 I 122

Cass., sez. I, 24.3.1982, n. 2042 (Min. beni culturali c. Sacerdoti), Foro it. 1982 III 285

= Foro amm. 1983 I 1610 = Rass.Cons.Stato 1982 I $356=$ Giust.civ. 1982, 2999

Trib. Torino 25.3.1982 (Repubblica dell'Ecuador, Casa della cultura ecuadoriana c. Danusso, Matta e altri), Riv.dir.int.priv.proc. 18 (1982) 625

Cons.Stato, sez. VI, 9.8.1986, n. 630 (Min. beni culturali c. Comune di Bordighera), Foro it. 1987 III 142

Cons.giust.sic., 25.3.1987, n. 78 (Lanucara c. Assessorato regionale Beni culturali e ambientali e Pubblica istruzione, Sopraintendente ai beni artistici e storici di Palermo), Foro amm. 1987 I 554

Trib. Roma 27.6.1987 (Stato francese c. Min.B.C.A. e De Contessini), Riv.dir.int. 71 (1988) 920; Dir.com.int. 2 (1988) 611; Riv.dir.int.priv.proc. 25 (1989) 652

T.A.R. Lazio, sez. II, 28.9.1987, n. 1516 (Soc. Kirzam c. Min.B.C.A.), Foro it. 1988 III 415

T.A.R. Veneto, sez. I, 1.2.1988, n. 47 (Mac Lean Bather c. Min.B.C.A.), Foro amm. 1989, 1086

Cons.Stato, sez. VI, 24.1.1989, n. 22 (Pagenstecher c. Provincia autonoma di Bolzano, Min.B.C.A. e altro), Foro amm. 1989 I 177

Cons.Stato, ad.gen., 13.7.1989, n. 59/89 (Min.B.C.A.), Foro it. 1990 III 356

C.Cost, 9.3.1990, n. 118 (Perugia ed altri - Min.B.C.A. ed altro - Presidente del Consiglio dei Ministri), Giur.cost. 1990, 660 = Racc.Uff. 685

Cons.Stato, sez. IV, 25.8.1990, n. 752 (Soc. Kirzam c. Min.B.C.A.), Rass.Cons.Stato 1990 I 1019 
Cons.Stato, ad.plen., 23.9.1991, n. 7 (Pagenstecher c. Provincia autonoma di Bolzano, Min.B.C.A. e altro), Rass.Cons.Stato 1991 I 1293 = Foro it. 1992 III 1 = Foro amm. 1992 I 2242, nota Cannada-Bartoli (744) = Riv.it.dir.pubbl.com 1992, 949, nota Roccella $(780)=$ Riv.dir.int.priv.proc. 29 (1993) 431

T.A.R. Lazio, sez. II, 16.4.1992, n. 1106 (The Isamu Noguchi Fondation Inc. ed altro c. Min.B.C.A.), TAR 1992 I 1779

Cons.Stato, sez. VI, 18.10.1993, n. 741 (Righi Parenti c. Min.B.C.A. e Ceccherini), Foro it. 1994 III 121

Cons.Stato, sez. VI, 30.10 .1993 , n. 787 (Pastega e altro c. Min.B.C.A.), Foro it. 1994 III 329 = Rass.Cons.Stato 1993 I 1325

Cass., sez. I, 24.11.1995, n. 12166 (Stato francese - Min.B.C.A. c. De Contessini e altri), Foro it. 1996 I $907=$ Riv.dir.int. 80 (1997) $515=$ Riv.dir.int.priv.proc. 33 (1997) 427

Cass., 9.9.1997, n. 8743, Giust.civ. 1998, 2650

Cass., sez. I, 28.8.1998, n. 8589 (Comune di Camerino c. Min.B.C.A.), Foro it. 1998 I 3167

Cons.Stato, sez. VI, 21.9.1999, n. 1243 (Comune di Zola Pedrosa c. Theodoli Braschi e altri), Foro it. 2000 III 17

Cons.Stato, sez. VI, 8.2.2000, n. 677 (Settimo Centro Società Consortile s.r.l. c. Min.B.C.A.), Giur.it. 2000, 1298

Cons.Stato, sez. VI, 24.1.2005, n. 106 (zsf. in: AEDON - Rivista di arti e diritto on line, n. 1, 2006)

Cons.Stato, sez. VI, 31.1.2005, n. 256 (zsf. in: AEDON - Rivista di arti e diritto on line, n. 1, 2006)

Cons.Stato, sez. VI, 22.3.2005, n. 1160 (zsf. in: AEDON - Rivista di arti e diritto on line, n. 1, 2006)

Cons.Stato, sez. VI, 21.10.2005, n. 5939 (zsf. in: AEDON - Rivista di arti e diritto on line, n. 1, 2006)

Cons.Stato, sez. VI, 17.2.2006, n. 659 (zsf. in: AEDON - Rivista di arti e diritto on line, n. 2, 2006)

\section{Frankreich}

Cons.d'Et. 2.6.1975 (Epoux Saint Arroman c. Réunion des musées nationaux et ministre des Affaires culturelles), Rec.Cons.d'Etat 1975, 795

Cons.d'Et. 27.3.1981 (Schlumpf), Rec.Cons.d'Etat 1981, 168

Court d'Appel de Montpellier 18.12.1984 (Ville de Genève et Fondation Abegg c. Consorts Margail), Rev.crit. 74 (1985) 559

Cons.d'Et. 17.6.1985 (Dauberville), Rec.Cons.d'Etat 1985, 184

Cons.d'Et. 7.10.1987 (Min. de la Culture c. Consorts Genty), Rec.Cons.d'Etat 1987, $305=$ R.D.S. 1988 I $269=$ AJDA 1987, 720

Court de Cassation, 15.4.1988 (Fondation Abegg et Ville de Genève c. Mme Ribes), Rev.crit. 78 (1989) 100 
Cons.d'Et. 24.1.1990 (Amon), Rec.Cons.d'Etat 1990, 13 = AIDA 1990, 423

Cass.Crim. 11.6.1990 (Grouet), R.D.S. 1990 II 206

Cons.d'Et. 31.7.1992 (Jacques Walter), Rec.Cons.d'Etat 1992, 313 = La Semaine Juridique 1993 II $22044=$ R.D.S. 1994 I 17

Cour d'appel de Paris 6.7.1994 (Jacques Walter), R.D.S. 1995 I 254

Tribunal administratif de Paris 30.6.1999 (Rodolphe Hottinger), J-Cl. Adm. 465-20, Ziff. 35

Tribunal administratif de Paris, 30.6.1999 (Germaine de Chaisemartin), J-Cl. Adm. 465-20, Ziff. 58

\section{Deutschland}

VGH München 22.3.1963, BayVB1. 1963, 254

BVerwG 28.5.1965, BVerwGE 21, 191 = NJW 1965, 1932

VG Gelsenkirche 5.6.1985, 4 K 1357/84, unveröffentlicht

VG Sigmaringen 25.11.1985, 1 K 313/84, unveröffentlicht

VGH Mannheim 14.3.1986, NJW 1987, 1440 (Glasmalerei, Elfenbeinskulptur)

VG Hannover 9.6.1989, NVwZ-RR 1991, 643 (Silberzimmer der Welfen)

VGH München 4.12.1991, NJW 1992, 2584 (Käfersammlung)

BVerwG 30.3.1992, NJW 1992, 2584 (Käfersammlung)

OVG Lüneburg 19.5.1992, NVwZ-RR 1993, 79 (Silberzimmer der Welfen)

BVerwG 27.5.1993, BVerwGE 92, 288 = NJW 1993, 3280 = DVB1. 1993, 1099 (Silberzimmer der Welfen)

BVerfG 2.3.1999, BVerfGE 100, 226 = NJW 1999, 2877

\section{Vereinigtes Königreich}

Attorney General of New Zealand v. Ortiz and others [1982] 1 Q.B. 349; [1982] 3 All E.R. (Q.B. and C.A.) 432; [1982] 3 W.L.R. (C.A.) 570; [1982] 2 W.L.R. (Q.B.) 10; [1983] 2 All E.R. (H.L.) 93; [1984] 1 A.C. 1 C.A. (H.L.)

Kingdom of Spain v. Christie, Manson \& Woods Ltd. [1986], 1 W.L.R. 1120 (Ch.D.) = [1986] 3 All E. R. 28 (Ch.D.)

The King of Italy and Italian Government v. Marquis Cosimo de Medici Tornaquinci, Marquis Averardo de Medici Tornaquinci and Christie, Manson and Woods, [1917/ 1918] 34 Times L.Rep. 623 (Ch.D.), auf italienisch übersetzt in: Riv.dir.int. 14 (1921/22) 194

\section{Schweiz}

BGE 118 Ia 384 ff. (Kinvar AG) 
LXIV Verzeichnis übernationaler und nationaler Quellen

\section{Vereinigte Staaten von Amerika}

Jeanneret v. Vichey, 541 F.Supp. 80 (S.D.N.Y. 1982) reversed and remanded 693 F. $2^{\text {nd }}$ 259 ( $2^{\text {nd }}$ Cir. 1982)

United States v. Hollinshead, 495 F. $2^{\text {nd }} 1154$ ( $9^{\text {th }}$ Cir.)

Autocephalous Greek Orthodox Church of Cyprus v. Goldberg \& Feldman Fine Arts Inc. 717 F.Supp. 1374 (S.D.Ind. 1989), 917 F. $2^{\text {nd }} 278$ ( $7^{\text {th }}$ Cir. 1990)

Dole v. Carter, 444 F.Supp. 1065 (D.Kan. 1977) und Dole v. Carter, 569 F. $2^{\text {nd }} 1109\left(10^{\text {th }}\right.$ Cir. 1977) 